\title{
Stopping intracellular leakage with chemistry
}

Researchers develop a strategy to improve the intracellular retention of fluorescent probes and thus their imaging sensitivity.

A colorful array of turn-on fluorescent probes is available for lighting up different molecules and observing their interactions in living cells. Specificity for the intended target and high sensitivity are top requirements in probe design. But many sensors, although highly sensitive and specific in vitro, do not perform well in living cells.

Tetsuo Nagano of the University of Tokyo knows this well. Over the years, his group has developed many fluorescent probes to image various cellular processes. But often, probes that worked well in the test tube did not translate effectively to cellular application. One explanation for poor performance is that the probe in question may be subject to leaking from the cells. "Intracellular retention is a big problem for a large number of fluorescent probes," says Nagano. His group hypothesized that by using chemistry to redesign probes to improve their intracellular retention, they could therefore also improve the sensitivity of fluorescence detection.

Fluorescein is an attractive scaffold for intracellular sensors because it has high quantum yield, it can be excited by visible light, it is soluble and it can readily be modified-but the downside is that it is poorly retained by cells. However, Nagano's group observed that calcein, a fluorescein derivative, was well retained by cells. Calcein has a unique structure: it contains two imino-diacetic acid groups, which the researchers thought could be responsible for calcein's good intracellular retention.

To test their hypothesis, they synthesized membrane-permeant derivatives of fluorescein containing either one or two iminodiacetic acid groups; two imino-diacetic acid groups were required for optimal intracellular retention. They then used this concept to develop novel fluorescent probes for imaging highly reactive oxygen species and nitric oxide. With these well-retained probes, they visualized very low levels of the target molecules in living cells over a relatively long period, which would have been nearly impossible to monitor using conventional probes.

Nagano believes that this chemical approach to prevent cellular leakage should be broadly applicable for probes based on fluorescein as well as on other scaffolds. "I think it should be a general strategy to increase the sensitivity in living cells," he says.

\section{Allison Doerr}

\section{RESEARCH PAPERS}

Izumi, S. et al. A simple and effective strategy to increase the sensitivity of fluorescence probes in living cells. J. Am. Chem. Soc. 131, 10189-10200 (2009). 